\title{
Power-Efficient Pulse Width Modulation DC/DC Converters with Zero Voltage Switching Control
}

\author{
Changbo Long, Sasank Reddy, Sudhakar Pamarti, Lei He \\ EE Dept., UCLA \\ longchb@synopsys.com,\{sasank,spamarti,Ihe\}@ee.ucla.edu
}

\author{
Tanay Karnik \\ Intel \\ tanay.karnik@intel.com
}

\begin{abstract}
This paper proposes a power-efficient PWM DC/DC converter design with a novel zero voltage switching (ZVS) control technique. The ZVS control is realized by an inner feedback loop which is implemented by simple digital circuitry between the input and output of the power transistors and achieves real-time zero voltage switching (ZVS) for various loading and device parameters with power efficiencies over $90.0 \%$. In addition, an outer feedback loop is used to ensure that the output precisely tracks a reference voltage level. We have also built the relationship between the output voltage ripple and the speed of the voltage comparators which has shown to introduce new low-frequency signals to the loops and cause significant output voltage ripples. Experiment results show that the output ripple could be reduced by $4 \mathrm{x}$ by carefully handling the generation and propagation of these low frequency signals.
\end{abstract}

\section{Categories and Subject Descriptors}

B.7.1 [Integrated Circuits]: Types and Design Styles

\section{General Terms}

Design.

\section{Keywords}

DC/DC conversion, zero voltage switching.

\section{INTRODUCTION}

Power consumption has become one of the most important issues in modern electronics due to increased complexity and speed of the system. In order to curb the effect of power on a system as a whole, multiple power domains have been proposed as an architecture scheme for low power design. To support multi-Vdd, an array of supply voltages need to be generated. DC/DC converters can be integrated on chip and convert the input voltage to different voltage levels internally. Recently, a great deal of research [1-5] has been devoted to improving the power efficiency and reducing the area cost of on-chip DC/DC converters. However, there are

Permission to make digital or hard copies of all or part of this work for personal or classroom use is granted without fee provided that copies are not made or distributed for profit or commercial advantage and that copies bear this notice and the full citation on the first page. To copy otherwise, to republish, to post on servers or to redistribute to lists, requires prior specific permission and/or a fee.

ISLPED'06, October 4-6, 2006, Tegernsee, Germany.

Copyright 2006 ACM 1-59593-462-6/06/0010 ...\$5.00. still many unsolved problems. For instance, the basic linear regulator and the charge-recycling voltage regulator are designs that have been looked at as candidates for on-chip integration because there are no filter elements. However, the relative low power efficiency, typically less than $80 \%$ [1], of these designs has limited their application.

In this paper, we propose design techniques and analysis to address the above problems for high frequency PWM buck converters. We first introduce a real-time ZVS technique which relies on a feedback loop as opposed to tuning device parameters to achieve ZVS during design time as in traditional methods [4]. Our experiment results show that the feedback mechanism guarantees ZVS under different loading and device parameters. Furthermore, using the real-time ZVS technique we are able to achieve power efficiencies over $90.0 \%$. We then study close loop design and analysis of PWM buck converters. Our experiment results show that output voltage ripple in a closed loop PWM buck converter can be reduced up to $4 \mathrm{x}$ by correctly analyzing and optimizing the sources that generate and propagate low frequency signals.

\section{BACKGROUND AND DESIGN OVERVIEW}

\subsection{Principles of PWM Buck Converters}

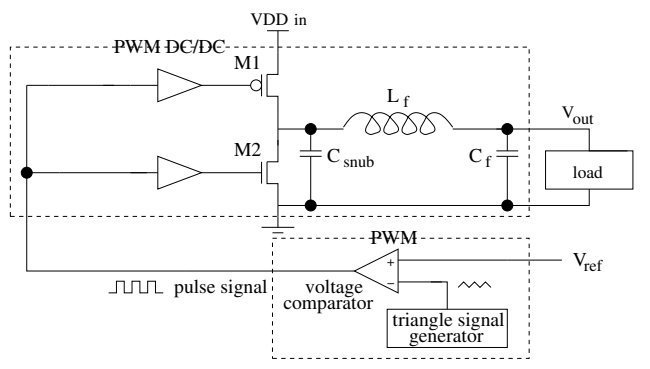

Figure 1: Schematic of a PWM buck converter.

Compared to other designs, such as linear regulators, PWM buck converters consume more area but have higher power efficiency. The schematic of a PWM buck converter is shown in Fig. 1. It consists of two power transistors, $M 1$ and $M 2$, with their drivers, a low-pass LC filter consisting of $L_{f}$ and $C_{f}$, a snubber capacitor $C_{s n u b}$, and a pulse width modulator.

The output voltage level $V_{\text {out }}$ is the DC component of the pulse signal generated by the PWM, and it is

$$
V_{\text {out }}=V d d_{\text {in }} \cdot D
$$

where $D$ is the duty cycle of the pulse signal, which is con- 
trolled by $V_{\text {ref }}$ as an input of the PWM. In fact,

$$
D=\frac{V_{r e f}}{V d d_{i n}} \text {. }
$$

Therefore, we have

$$
V_{\text {out }}=V_{\text {ref }} \text {. }
$$

As shown in [6], the output voltage ripple of a PWM buck converter can be expressed as

$$
\Delta V_{\text {out }}=\frac{V d d_{\text {in }}(1-D) D}{8 L_{f} C_{f} f^{2}},
$$

where $L_{f}$ and $C_{f}$ are the inductance and capacitance of the LC filter and $f$ is the frequency of the pulse signal. $f$ is also called the operation frequency of the buck converter.

Equation (4) shows that to keep $\Delta V_{\text {out }}$ at a low level, $L_{f}$ and/or $C_{f}$ has to be large if the operation frequency $f$ is low. In other words, an effective way to reduce the area of the LC filter in the buck converter is to use a high operation frequency [2]. However, a high operation frequency leads to a high switching power loss. To reduce the switching power loss, a technique called zero voltage switch (ZVS) has been widely adopted. As shown in [4], ZVS ensures that both power transistors switch under a zero voltage drop between source and drain.

\subsection{Overview of the Proposed Topology}

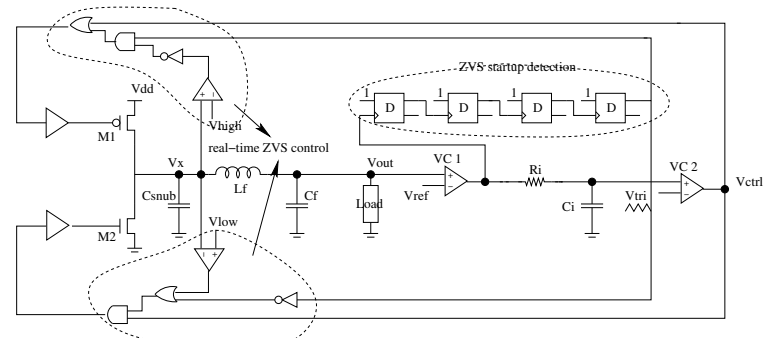

Figure 2: Overview of the proposed circuit topology.

Our study in this paper is based on the circuit topology shown in Figure 2. This circuit contains two feedback loops. The outer loop consists of two power transistors $M_{1}$ and $M_{2}$, LC filter $L_{f}$ and $C_{f}$, voltage comparator $V C 1, R C$ integrator $R_{i}$ and $C_{i}$, pulse modulation element $V C 2$, and real-time ZVS circuitry. The inner loop starts from the output of the two power transistors, passes through the ZVS circuitry and ends at the input of the two power transistors.

The outer loop ensures that the output voltage level $V_{\text {out }}$ tracks the reference voltage level $V_{r e f}$. It is considered a negative feedback loop since there are only one set of negative components, the power transistors, in the entire looop. For example, if $V_{\text {out }}$ is higher than $V_{\text {ref }}$, the output of voltage comparator $V C 1$ is high, $C_{i}$ is charged, and the voltage level at the positive input of $V C 2$ is increased, which increases the duty cycle of the switching signal and therefore decreases $V_{\text {out }}$.

The design of voltage comparator is adopted from [7], which has shown high resolution and low power consumption. The topology of the voltage comparator is shown in Figure 3, which is composed by an input amplification stage, two flip flops and a RS latch. Two clock signals are used to clear up previous results and evaluations. More details are described in [7].

The real-time ZVS technique is achieved by the inner feedback loop. To avoid the influence of startup strike, a detection sub-circuit is included as shown in Figure 2. The idea is not to startup the ZVS circuitry until $V_{\text {out }}$ has been stabilized. More details will be described in Section 4 .

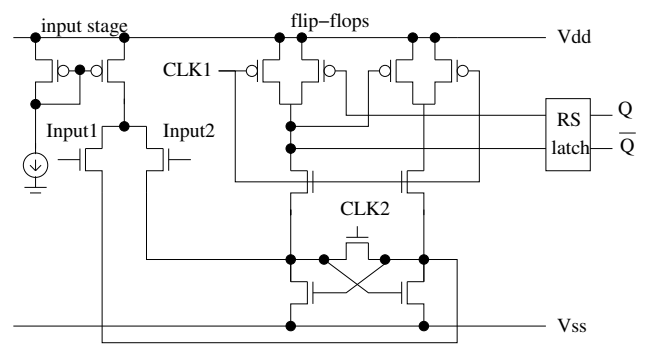

Figure 3: Topology of the voltage comparator.

\section{CLOSE LOOP DESIGN AND ANALYSIS}

\subsection{Analysis on output voltage ripple}

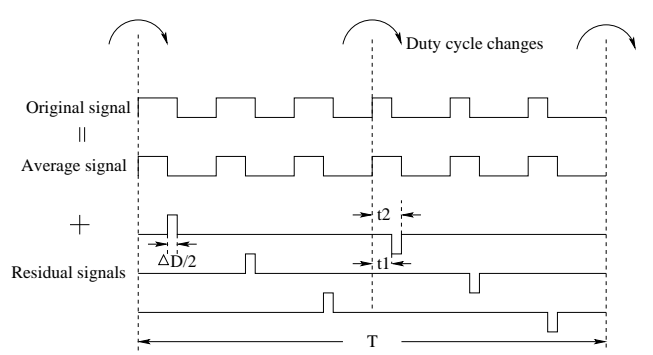

Figure 4: Illustration of changes in duty cycle in the switching signal.

Our study is based on the design shown in Figure 2. The voltage comparator $V C_{2}$ in the figure modulates the duty cycle of the switching signal feeding the two power transistors and this modulation introduces a signal that contains a wide range of frequencies. Low frequency components of this signal may pass the $L C$ filter and cause a voltage ripple with a frequency approximately equal to the frequency of the $L C$ filter, $1 /\left(2 \pi \sqrt{L_{f} C_{f}}\right)$, at the output terminal. Note that the voltage comparator $V C_{1}$ may also bring in low frequency components but it is filtered by the $R_{i} C_{i}$ integrator and has a smaller impact to the overall output ripple.

Figure 4 illustrates the behavior of the operating switching signal during steady state. In the figure, this signal is decomposed into an average signal, which maintains a constant duty cycle and a set of residual signals. The average signal contains only high frequency components, and the residual signals contain a wide range of frequency components. The duty cycle switches between two states $D_{1}$ and $D_{2}$ has a frequency the same as the output voltage ripple, which is roughly equal to frequency of the $L C$ filter, i.e.,

$$
T=2 \pi \sqrt{L_{f} C_{f}} .
$$

By Fourier transformation, the residual signals can be expressed as follows,

$$
\begin{aligned}
f(t)= & \frac{2 V_{d d}}{\pi}\left[\sum_{n=0}^{\infty}\left(\frac{\sin (2 n+1) \omega t_{2}-\sin (2 n+1) \omega t_{1}}{2 n+1}\right) \cos (2 n+1) \omega t\right. \\
& \left.+\sum_{n=0}^{\infty}\left(\frac{\cos (2 n+1) \omega t_{1}-\cos (2 n+1) \omega t_{2}}{2 n+1}\right) \sin (2 n+1) \omega t\right] \\
= & \frac{2 V_{d d}}{\pi} \sum_{n=0}^{\infty} \frac{\sqrt{2-2 \cos (2 n+1) \omega\left(t_{2}-t_{1}\right)}}{2 n+1} \cos ((2 n+1) \omega t+\phi) \\
\approx & \frac{2 V_{d d}}{\pi} \sum_{n=0}^{\infty} \omega\left(t_{2}-t_{1}\right) \cos ((2 n+1) \omega t+\phi),
\end{aligned}
$$


where

$$
\omega=\frac{1}{\sqrt{L_{f} C_{f}}}
$$

Notice that $t_{2}-t_{1}=T \cdot \frac{\Delta D}{2}$, we have

$$
f(t)=\frac{V_{d d} T}{\pi \sqrt{L_{f} C_{f}}} \Delta D \sum_{n=0}^{\infty} \cos ((2 n+1) \omega t+\phi) .
$$

From (6) we can see that $\omega$ is the $3 \mathrm{db}$ frequency of the $L C$ filter which implies that the magnitude of a signal at this frequency becomes half when it passes through the $L C$ filter. Also, equation (7) implies that the residual signals contain frequency components of $\omega, 3 \omega, \cdots$, etc and the magnitudes of these components are proportional to $\Delta D$. Given that $\omega$ is the $3 \mathrm{db}$ frequency of the $L C$ filter, when the residual signals enter into the $L C$ filter, the low frequency components, such as $\omega, 3 \omega$ and $5 \omega$, can pass through the $L C$ filter with significant magnitudes and appear as a ripple at the output terminal. Because the magnitudes of the low frequency components of $\omega, 3 \omega$ and $5 \omega$ etc. are proportional to $\Delta D$, the magnitude of the ripple is proportional to $\Delta D$ too. Practically every voltage comparator has a smallest value of $\Delta D$. In slower voltage comparators this $\Delta D$ are generally larger than in faster comparators. Therefore, we suggest to use fast voltage comparators to avoid large ripple at the output.

\section{REAL-TIME ZVS TECHNIQUE}

\subsection{Traditional ZVS techniques}

A representative traditional design-time ZVS technique without feedback is presented in [4]. To illustrate the ZVS conditions, each cycle of the internal switching signal is divided into four time fragments, $T_{1}, T_{2}, T_{3}$ and $T_{4}$ in the figure. During $T_{1}$ and $T_{3}$, the NMOS power transistor $M_{2}$ and PMOS power transistor $M_{1}$ close, respectively. During $T_{2}$ and $T_{4}$, both transistors are open. These two time fragments are called deadtimes. $V_{x}$ drops to zero at the beginning of $T_{1}$ when $M_{2}$ starts to close. Also, $V_{x}$ reaches Vdd when $M_{1}$ starts to close. Thus, $M_{1}$ and $M_{2}$ switch at a zero voltage drop between the source and drain i.e, zero voltage switching. During $T_{2}$, the inductance current $I_{L_{f}}$ charges $C_{\text {snub }}$ and $V_{x}$ increases to $V d d$. Similarly during $T_{1}$, the inductance current $I_{L_{f}}$ discharges $C_{\text {snub }}$ and $V_{x}$ decreases to zero.

One can see that the conditions to achieve ZVS are very restrictive. Parameters have to be re-tuned when loading current changes. Design-time ZVS techniques without feedback are vulnerable to variations of devices and loading.

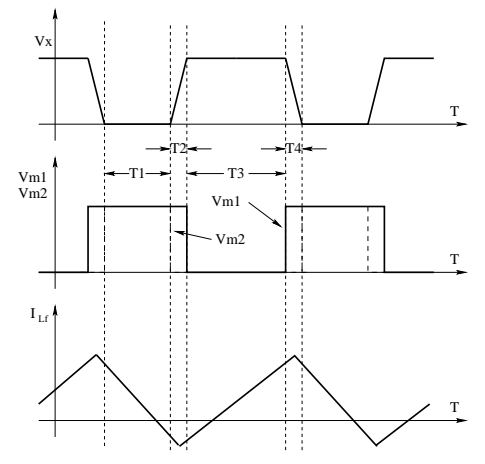

Figure 6: Conditions to achieve zero voltage switching.

\subsection{Real-time ZVS technique}

Assuming $V_{c t r l}$ as the control signal of the power transistors, without considering ZVS, the control signals for the PMOS power transistor, $M_{1}$, and NMOS power transistor, $M_{2}$, are the same as $V_{c t r l}$, i.e.,

$$
V_{m 1}=V_{m 2}=V_{c t r l}
$$

To achieve ZVS, $V_{m 1}$ and $V_{m 2}$ can be expressed as

$$
V_{m 1}=V_{c t r l}+\overline{V_{x}}
$$

and

$$
V_{m 2}=V_{c t r l} \cdot \overline{V_{x}},
$$

where $V_{x}$ is the output voltage level of the power transistors. Equation (9) ensures that $V_{m 1}$ is 1 whenever $V_{x}$ is 0 , which implies that $M_{1}$ turns on $\left(V_{m 1}\right.$ changes from 1 to 0$)$ only when $V_{x}$ is 1, i.e., the voltage drop between the source and drain of $M_{1}$ is zero. Similarly, (10) ensures that $V_{m 2}$ is 0 whenever $V_{x}$ is 1 , which implies that $M_{2}$ turns on $\left(V_{m 2}\right.$ changes from 0 to 1 ) only when $V_{x}$ is 0 , i.e., the voltage drop between the source and drain of $M_{2}$ is zero.

Equation (9) and (10) explain the principle to achieve ZVS. The implementation of this principle, however, needs more careful analysis. To ensure that $V_{x}$ stays at the perfect Vdd/zero level when $M_{1}$ and $M_{2}$ start to open, we use voltage comparators instead of inverters to implement $\overline{V_{x}}$ as shown in Figure 2. To drive $M_{1}, V_{x}$ is compared with $V_{\text {high }}=V D D-\Delta v$ and only when $V_{x}$ is is higher than $V_{\text {high }} M_{1}$ can open. Similarly, to drive $M_{2}, V_{x}$ is compared with a low voltage level $V_{\text {low }}=\Delta v$ and only when $V_{x}$ is lower than $V_{\text {low }}, M_{2}$ can open. In order to reduce the overshoot on $V_{x}$, we use a $\Delta v$ slightly larger than zero $(0.3 \mathrm{v})$. However, as shown in our experiment results, it is impossible to fully eliminate overshoot.

Overall, the real-time ZVS scheme presented as part of this design is very unique in the fact that it does not rely on manually calculating the duty cycle delays for a particular design. Although there are designs that do automatic ZVS control, they do not meet the power and area requirements for on-chip high frequency applications $[8,9]$.

\subsection{ZVS startup detection}

Initially, when the circuit is cold started, there is a period of time where the output signals have abnormally large variation. During this startup period there is no need to turn on the automatic ZVS calibration control for the system. In order to avoid tuning ZVS during the startup period, a series of D flip-flops are used as delay elements to prevent the startup of the ZVS calibration scheme until after the initial transient spikes, as shown in Fig. 2.

\section{EXPERIMENT RESULTS}

\subsection{Close loop design and analysis}

To verify the idea that the output voltage ripple is proportional to the smallest duty cycle change $\Delta D$, we have implemented the voltage comparator, as in Figure 3, at two different clock rates. One voltage comparator operates at $400 \mathrm{MHz}$ and the other at $1.6 \mathrm{GHz}$ frequencies. We compare the output voltage ripple of the PWM buck converters implemented with these two comparators in Figure 5. Note that $130 \mathrm{~nm}$ technology is used, the $V d d$ voltage is 1.3 Volt, and the reference voltage level is 0.85 Volt. As shown in the figure, the voltage ripple of the $400 \mathrm{MHz}$ comparator is about $11.7 \%$ as compared to the reference voltage, while the voltage ripple of the $1.6 \mathrm{GHz}$ comparator is $2.9 \%$. By increasing the frequency of the comparator by $4 \mathrm{X}$, we notice a reduction in ripple by around $4 \mathrm{X}$. These experiment results show that when designing the closed loop PWM buck converter, the elements of the closed loop need to be carefully considered so that the output voltage ripple is minimized. 


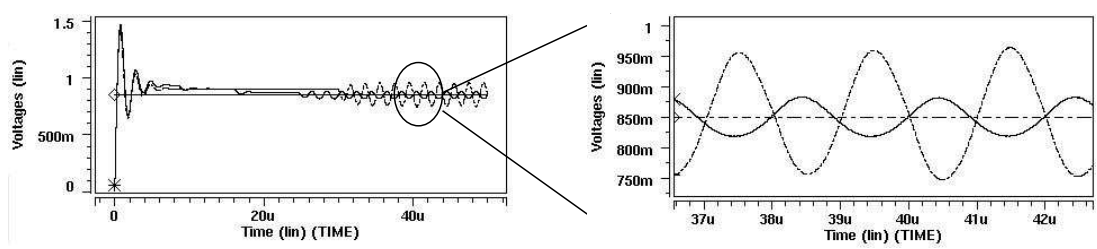

Figure 5: Comparison of voltage ripple between buck converters implemented by a $0.4 \mathrm{GHZ}$ voltage comparator and $1.6 \mathrm{GHz}$ voltage comparator.

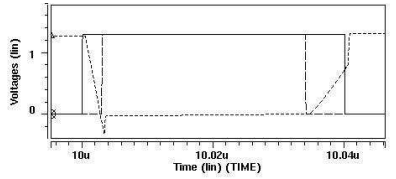

(a)

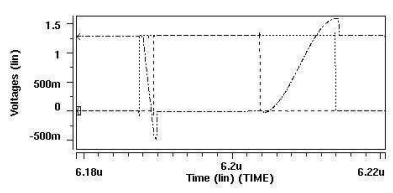

(d)

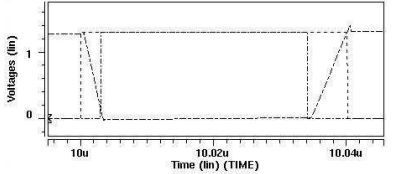

(b)

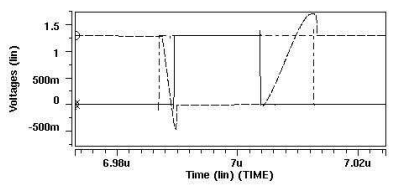

(e)

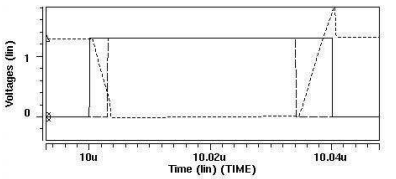

(c)

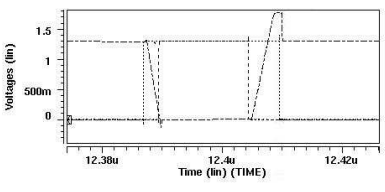

(f)

Figure 7: Design-time ZVS under loading of 5 ohms (a), 10 ohms (b), and 50 ohms (c) and real-time ZVS with feedback loop under loading of $5 \mathrm{ohms} \mathrm{(d),} 10 \mathrm{ohms} \mathrm{(e),} \mathrm{and} 50$ ohms (f). (A) and (c) fails ZVS.

\subsection{ZVS control}

One of the strong points of the design presented in this paper is the fact that the power of the transistors is consistent over a multitude of loads. This is mainly due to the fact that there is a real-time ZVS control system established in the circuit.

Figure 7 contains a comparison between design-time ZVS without feedback and real-time ZVS with feedback. In the case of the load of 5 and 50 ohms with design-time ZVS, one can see that the circuit is out of the ZVS mode of operation. With a load of $5 \mathrm{ohms}$, the $C_{\text {snub }}$ capacitor is charged too slow and the voltage of $V_{x}$ does not transition from the lower state to the higher state before the $M_{1}$ transistor switches on. On the other hand with a load of 50 ohms, the designtime ZVS circuit discharges the $C_{\text {snub }}$ too slowly and the circuit is in a non optimal energy efficiency state once again. The real-time circuit always stays in the ZVS mode of operation with the various loads. Although, there is an overshoot (refer to Section 4.2) for the real-time ZVS circuit, this does not affect the energy usage of the power transistors.

Overall, not having a proper ZVS control system will cause problems in terms of power usage of the switch transistors. In terms of overall power efficiency of the circuit, both ZVS operation and the actual magnitude of the current through the load play significant roles. But our experiments show that if the proper load current is used with real-time ZVS, power efficiencies are greater than $90 \%$ consistently for various loads.

\section{CONCLUSION}

In this paper, we have designed a novel PWM circuit that can be used to provide a range of Vdd levels for a variety of loads by two feedback loops. With the use of an inner feedback loop between the output and input of the power transistors, we are able to ensure real-time zero voltage switching. This enables the reduction of power consumed by these transistors and achieves power efficiencies over $90 \%$ for a va- riety of loads. Also, an outer feedback loop is employed in the PWM circuit to track the reference voltage level. We show that this closed loop should be propriately modeled and designed to ensure a low output voltage ripple.

\section{REFERENCES}

[1] S. Rajapandian, Z. Xu, and K. Shepard, "Charge-recycling voltage domains for energy-efficient low-voltage operation of digital cmos circuits," in Computer Design, 2003. Proceedings. 21st International Conference on, pp. 98-102, 2003.

[2] V. Kursan, S. G. Narendra, V. K. De, and E. G. Friedman, "Analysis of buck converters for on-chip integration with a dual supply voltage microprocessor," IEEE Trans. VLSI Syst., vol. 11, pp. 514-522, June 2003.

[3] G. Schrom, P. Hazucha, J.-H. Hahn, V. Kursun, D. Gardner, S. Narendra, T. Karnik, and V. De, "Feasibility of monolithic and 3d-stacked dc-dc converters for microprocessors in 90nm technology generation," in Proc. Intl. Symp. Low Power Electronics and Design, pp. 263-268, 2004.

[4] A. J. Stratakos, S. R. Sanders, and R. W. Brodersen, "A low-voltage cmos dc-dc converter for a portable battery-operated system," in Power Electronics Specialists Conference, PESC' 94 Record., 25th Annual IEEE, pp. 619-626, June 1994.

[5] A. J. Stratakos, "High-efficiency low-voltage dc-dc conversion for portable applications," PhD dissertation, University of California, Berkeley, 1998.

[6] D. W. Hart, ed., Introduction to Power Electronics. Prentice Hall, Upper Saddle River, N.J., 1997.

[7] G. Yin, F. Eynde, and W. Sansen, "A high-speed CMOS comparator with 8-b resolution," IEEE Journal of Solid-state Circuits, vol. 27, pp. 208-211, Feb. 1992.

[8] Y. Jang and M. Jovanovic, "A new zvs-pwm full-bridge converter," in IEEE International Telecommunications Energy Conference, pp. 232-239, Septemeber 2002.

[9] J. Cho, J. Sabate, G. Hua, and F. Lee, "Zero-voltage and zero-current-switching full bridge pwm converter for high power application," in IEEE Transactions on Power Electronics, pp. 102-108, May 1994. 